\title{
Concurrent Use of Prescription Drugs and Herbal Medicinal Products in Older Adults: A Systematic Review
}

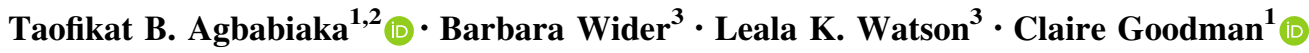

Published online: 1 December 2017

(c) The Author(s) 2017. This article is an open access publication

\begin{abstract}
Background The use of herbal medicinal products (HMPs) is common among older adults; however, little is known about concurrent use with prescription drugs, as well as potential interactions associated with such combinations. Objective The aim of this systematic review was to identify and evaluate the literature on concurrent prescription and HMP use among older adults to assess prevalence, patterns, potential interactions and factors associated with this use.

Methods Systematic searches were conducted in the MEDLINE, PsycINFO, EMBASE, CINAHL, AMED, Web of Science and Cochrane databases from inception to May 2017 for studies reporting concurrent use of prescription medicines with HMPs in adults $\geq 65$ years of age. Quality was assessed using the Joanna Briggs Institute checklists.
\end{abstract}

Electronic supplementary material The online version of this article (https://doi.org/10.1007/s40266-017-0501-7) contains supplementary material, which is available to authorized users.

Taofikat B. Agbabiaka

t.b.agbabiaka@herts.ac.uk

Barbara Wider

B.Wider@exeter.ac.uk

Leala K. Watson

L.K.Watson@exeter.ac.uk

Claire Goodman

c.goodman@herts.ac.uk

1 Centre for Research in Primary and Community Care (CRIPACC), University of Hertfordshire, Hatfield, UK

2 Patient Safety, Medical Directorate, NHS Improvement, London, UK

3 Institute of Health Research, University of Exeter Medical School, Exeter, UK
And the Evidence for Policy and Practice Information and Co-ordinating Centre (EPPI-Centre) three-stage approach to mixed method research was used to synthesise data.

Results Twenty-two studies were included. A definition of HMPs or what was considered an HMP was frequently missing. Prevalence of concurrent use by older adults varied widely between 5.3 and $88.3 \%$. Prescription medicines most combined with HMPs were antihypertensive drugs, $\beta$-blockers, diuretics, antihyperlipidemic agents, anticoagulants, analgesics, antihistamines, antidiabetics, antidepressants and statins. The HMPs most frequently used were Ginkgo biloba, garlic, ginseng, St John's wort, Echinacea, saw palmetto, evening primrose oil and ginger. Potential risks of bleeding due to the use of Ginkgo biloba, garlic or ginseng with aspirin or warfarin was the most reported herb-drug interaction. Some data suggest being female, and having a lower household income and less than a high-school education were associated with concurrent use.

Conclusion The prevalence of concurrent prescription drugs and HMP use among older adults is substantial and potential interactions have been reported. Knowledge of the extent and manner in which older adults combine prescription drugs will aid healthcare professionals in appropriately identifying and managing patients at risk. Systematic Review Registration Number: PROSPERO 2014:CRD42014009091. 


\section{Key Points}

Concurrent use of prescription drugs and herbal medicinal products (HMPs) among older adults is substantial, with prevalence varying widely between 5.3 and $88.3 \%$.

The most commonly combined prescription medicines were antihypertensive drugs, $\beta$-blockers, diuretics, anticoagulants, analgesics, antidiabetics, antidepressants and statins. And the most frequently used HMPs were Ginkgo biloba, garlic, ginseng, St John's wort, Echinacea, saw palmetto, evening primrose oil and ginger.

There is still limited knowledge of the extent and manner in which older adults combine prescription drugs with HMPs.

\section{Introduction}

The world population is ageing. According to the World Health Organization (WHO), by 2050 the population of people aged $\geq 60$ years will double and around 400 million people will be $\geq 80$ years of age [1]. By 2040, nearly one in four people $(24.2 \%)$ in the UK will be aged 65 years or older [2]. With pharmacotherapy facilitating an ageing population [3], older populations rely on complex polypharmacy to manage chronic health conditions [4]. Older adults are the biggest consumers of prescription and over-the-counter (OTC) medicines [5-7], and it is also well-recognised that self- medication [8, 9] and consumption of non-prescription medicines, particularly herbal and other dietary supplements, is widespread among older adults [10-16]. Polypharmacy [17-19] increases the risks of adverse drug reactions (ADRs) and interactions [20, 21]. With healthcare systems increasingly burdened with more hospitalisations and prolonged hospital stays due to ADRs [22], potential herb-drug interactions are major clinical and economic concerns.

In the UK, prescriptions dispensed for those aged over 60 years accounted for $51.2 \%$ of the total net cost for all prescriptions in 2014 [23]. In addition, up to one-quarter of adults use herbal medicinal products (HMPs) [6, 24, 25]medicinal products where the active ingredients consist exclusively of herbal substances or herbal preparations [26]. HMPs are covered by Directive 2001/83/EC on the Community code relating to medicinal products for human use ("Directive on human medicinal products") [26]. They are mostly bought over the counter, by self-prescription, and are generally not disclosed to healthcare practitioners $[6,24,25]$. ADRs could occur due to interactions between conventional drugs and HMPs, some of which may have serious consequences [15, 27-29]. For example, St. John's wort (Hypericum perforatum) taken with serotonin reuptake inhibitors increases the risk of serotonin syndrome in older adults [27]. Despite concerns of possible harmful interactions, little is known about the concurrent use of these medicines by older adults.

The aim of this systematic review was to identify and evaluate the literature on concurrent prescription and HMP use among older adults to assess (1) prevalence, (2) patterns, (3) potential interactions and other safety risks, and (4) factors associated with this use.

\section{Methods}

The full review protocol has previously been published [30]. This review was conducted according to the principles of systematic review [31] and is reported following the Preferred Reporting Items for Systematic Reviews and Meta-Analyses (PRISMA) guidelines [32].

\subsection{Eligibility Criteria}

Literature searches identified studies assessing the prevalence and patterns of concurrent HMPs or herbal dietary supplements used with prescription medicines. Cross-sectional studies, case reports and case series were included. However we excluded $\mathrm{PhD}$ theses, editorials, commentaries, in vitro experiments and animal studies. Studies assessing herbal medicine as part of a therapeutic system or system of medicine such as traditional Chinese medicine, Ayurveda, Kampo, Siddha, Unani and homeopathic herbal remedies were also excluded from the review. As were studies assessing the concurrent use of vitamins, minerals and non-herbal dietary supplements or combination products containing herbal and non-herbal substances with prescription medicines.

The WHO defined 'elderly' as individuals over the age of 65 years in developed countries, and over 60 years in developing countries. For the purpose of this review, we have adopted the minimum age of 65 years since the majority of studies identified from our literature searches were conducted in developed countries. Therefore, studies with participants aged 65 years or older, studies with a mean participant age $\geq 65$ years, or studies from which data for participants aged $\geq 65$ years could be extracted were included in this review. 


\subsection{Search Methods for Identification of Studies}

The following databases were searched until May 2017: Cumulative Index to Nursing and Allied Health Literature (CINAHL) via EBSCO, Cochrane Library, Excerpta Medica database (EMBASE) via OVID, MEDLINE via OVID, the Allied and Complementary Medicine Database (AMED) via EBSCO, PsycINFO via OVID, and Web of Science. Medical Subject Headings (MeSH) and text words included 'herbal medicine', 'prescription drugs' and 'aged'. The scientific names and common names of herbs most documented for concurrent use were applied to ensure a broad search strategy.

No restrictions were placed on language of publication, and reference lists of all identified studies were checked for relevant studies not identified by the electronic searches. Lateral searches were also conducted using the related citation function in PubMed and cited by function in Google Scholar to capture all relevant articles. The search strategy is available as electronic supplementary Appendix S1.

\subsection{Data Collection and Analysis}

\subsubsection{Selection of Studies}

Retrieved references from all the databases were downloaded into Endnote files and then merged. All duplicate studies were recorded before discarding. Two reviewers (TA and BW) scanned all titles and abstracts for potential relevance. Any article for which there was uncertainty about relevance was retained and the full text assessed. Using a predesigned eligibility checklist, two reviewers (TA and BW) independently assessed full-text articles against the eligibility criteria and recorded an eligibility code. Studies that did not meet the inclusion criteria were excluded and the reasons recorded. Disagreements on eligibility were resolved through discussions between the two reviewers (TA and $\mathrm{BW}$ ), and the third reviewer (CG) was consulted if no consensus was reached. Full texts of all articles that met the eligibility criteria were obtained and downloaded into Endnote.

\subsubsection{Data Extraction and Management}

A data extraction form was designed for the review, then piloted and amended to ensure that all the required information could be extracted. Data from individual studies were extracted by the first reviewer (TA) using this form, and validated by the second reviewer (BW). Key information extracted included:

- Publication details: authors, year of publication, country in which the study was conducted.

- Study design: study type, recruitment and data collection method.

- Participants: demographic and socioeconomic characteristics, sampling and sample size, previous medical diagnosis, etc.

- Primary outcomes: prevalence of concurrent use, name and number of HMPs and prescription drugs, pattern of use, and number and types of adverse reactions or potential interactions.

- Secondary outcomes: disclosure, satisfaction or dissatisfaction, and cost expended on HMPs.

- Study limitations: response bias, selection bias, representativeness of sample, etc.

\subsubsection{Quality Assessment of Included Studies}

The Joanna Briggs Institute (JBI) checklists for appraising studies reporting prevalence data [33] and for case reports [34] were used to screen selected studies prior to inclusion in the review. Two reviewers independently assessed each of the included studies against the criteria on the JBI checklist to minimise bias and establish methodological validity. The JBI checklist for prevalence studies was the preferred assessment tool because it can be used across different study designs reporting prevalence. The checklist also addresses issues of internal and external validity critical to prevalence data. Any disagreements between reviewers were resolved through discussion.

\subsubsection{Data Synthesis}

We used the Evidence for Policy and Practice Information and Co-ordinating Centre (EPPI-Centre) three-stage approach to mixed method research to synthesise data [35]. A first synthesis was conducted to address the prevalence, pattern of use and patient characteristics associated with concurrent use of HMPs and prescription medicines. The second synthesis focused on safety issues and other factors associated with concurrent use, i.e. disclosure, satisfaction and cost/resources. Finally, using thematic synthesis, we identified key themes and commonalities. Findings were summarised as a narrative account addressing each of the review questions. A detailed discussion of the limitations of the included studies and the implications of our findings was also provided. 
Fig. 1 Study selection process

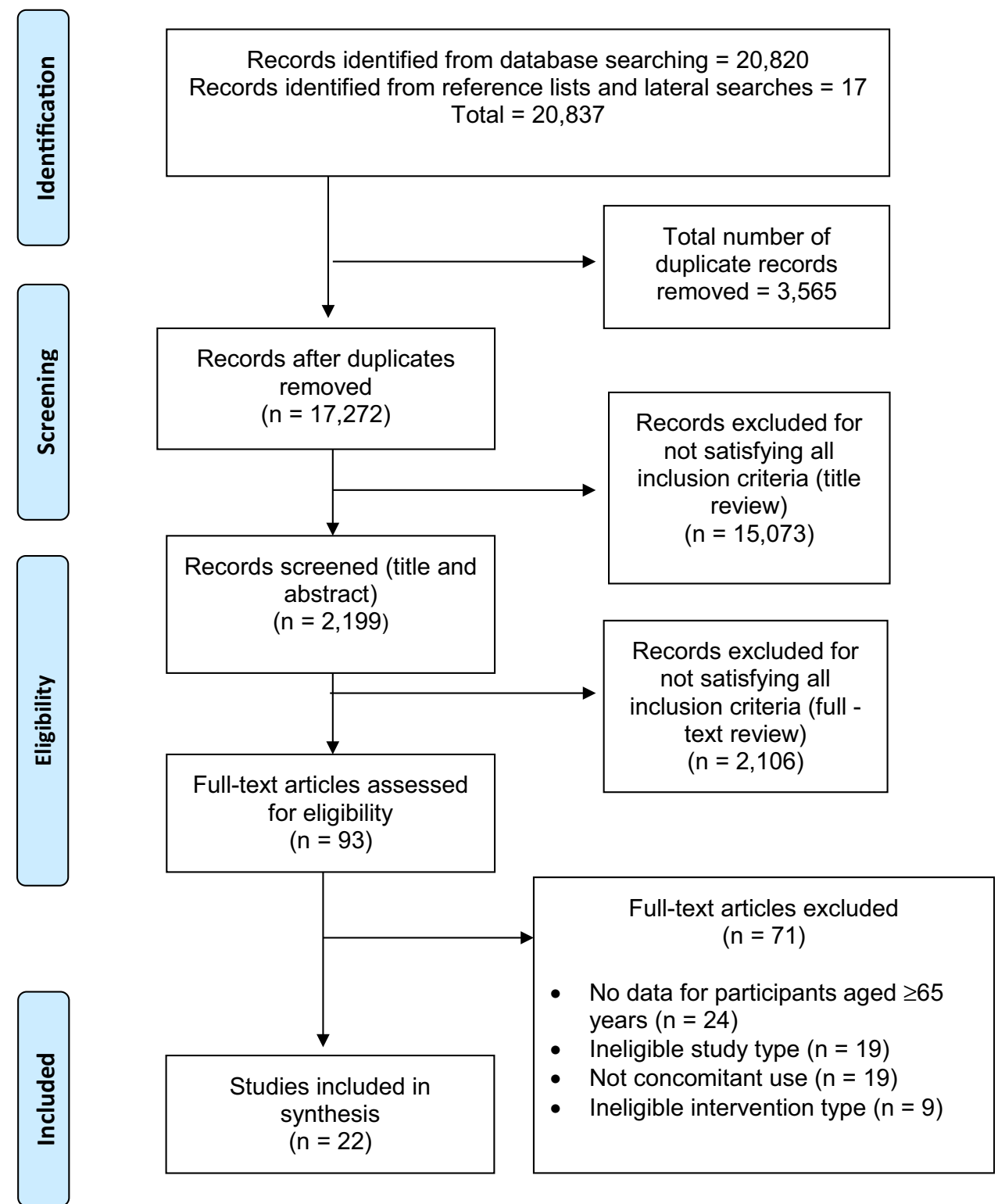

\section{Results}

\subsection{Results of the Search}

The literature searches identified 20,837 titles and abstracts. Initial screening of titles and abstracts identified 2199 potentially relevant articles; a total of 2106 articles were excluded for not satisfying all the inclusion criteria. Full texts of the remaining 93 articles were obtained to assess for eligibility. At the end of the eligibility process, 71 articles were excluded for the following reasons: type of intervention (e.g. non-herbal combinations, non-oral; $n=9)$, age $(n=24)$, study type $(n=19)$, and no concomitant use $(n=19)$. Twenty-two studies met our inclusion criteria and were included in this systematic review (Fig. 1).

\subsection{Study Characteristics}

Table 1 is a summary of included studies, providing information on study setting, sample characteristics, prevalence of concurrent use, and most reported prescription medicines and HMPs, as well as interactions or potential interactions reported from such combinations.

All included studies were published in the English language, except one study published in Spanish [36]. Thirteen of the included studies were conducted in the USA [5, 17, 37-46, 54], two in Canada [47, 48] and two in the UK [16, 49]. Only one study each was conducted in Ireland [50], Norway [51], Turkey [52], Spain [36] and Jamaica [53]. The majority of studies $(n=16)$ were described as cross-sectional [5, 17, 36, 38, 40-42, 44, 45, 47-53], eight of which identified concurrent use of prescriptions with 


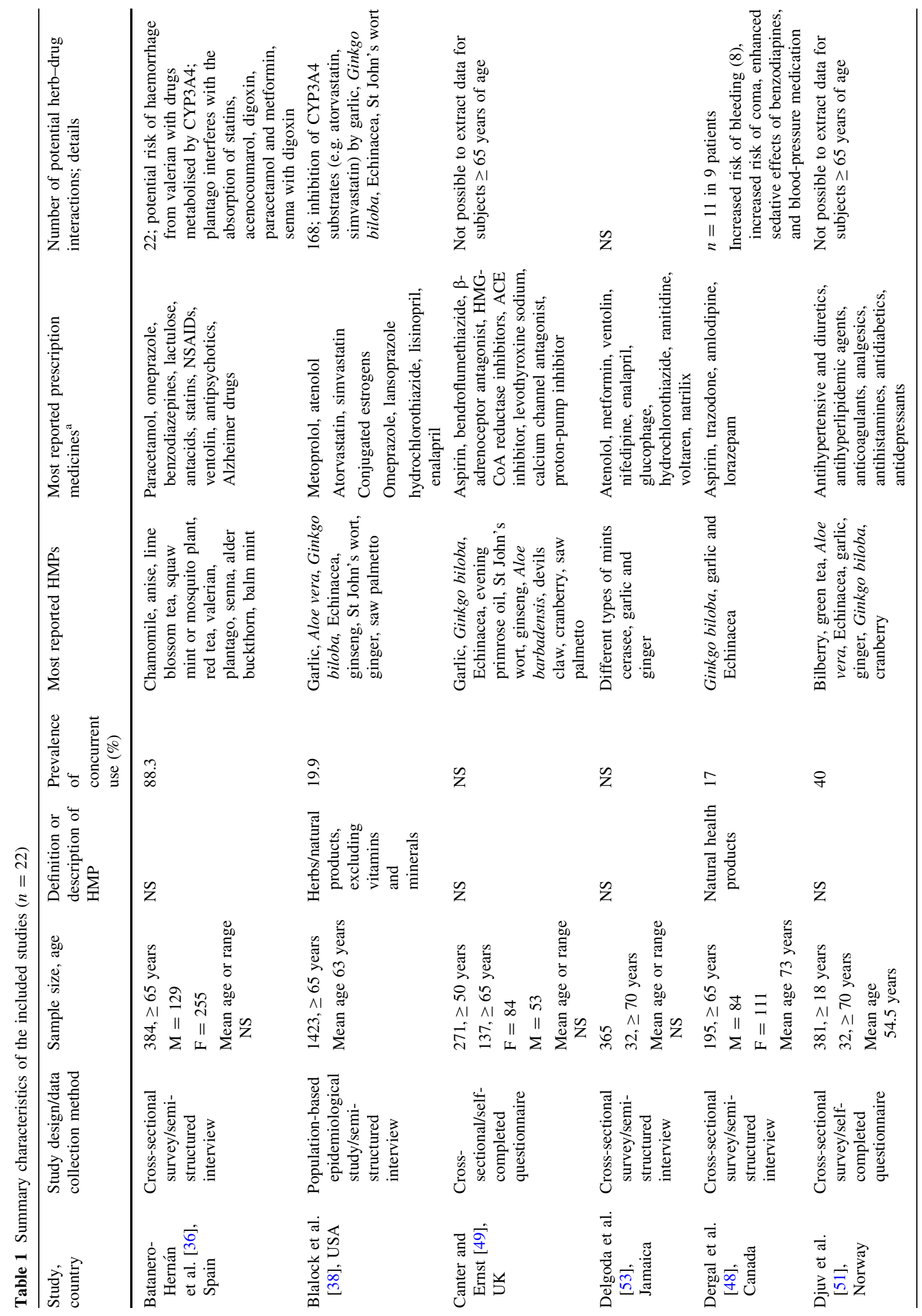




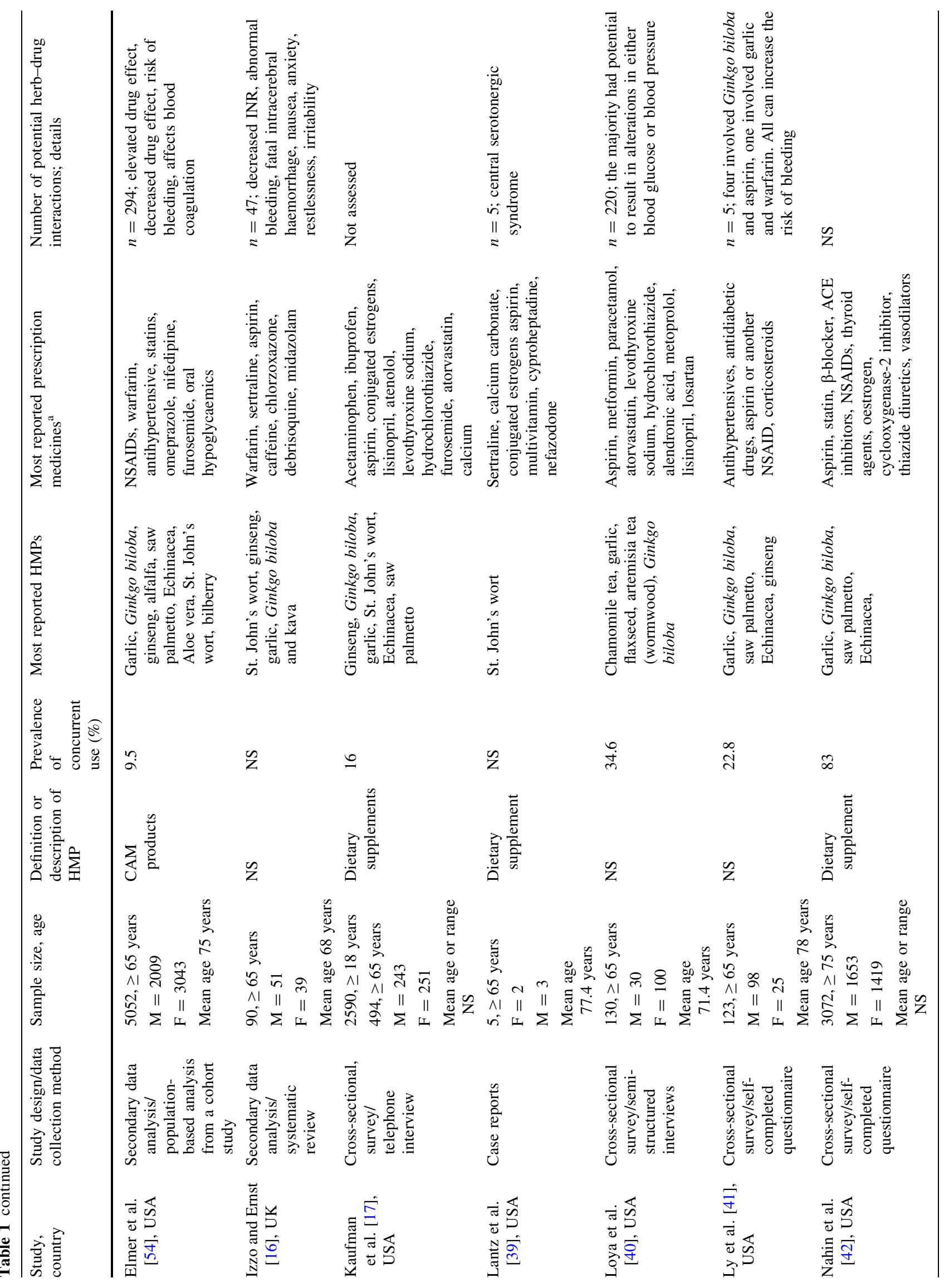




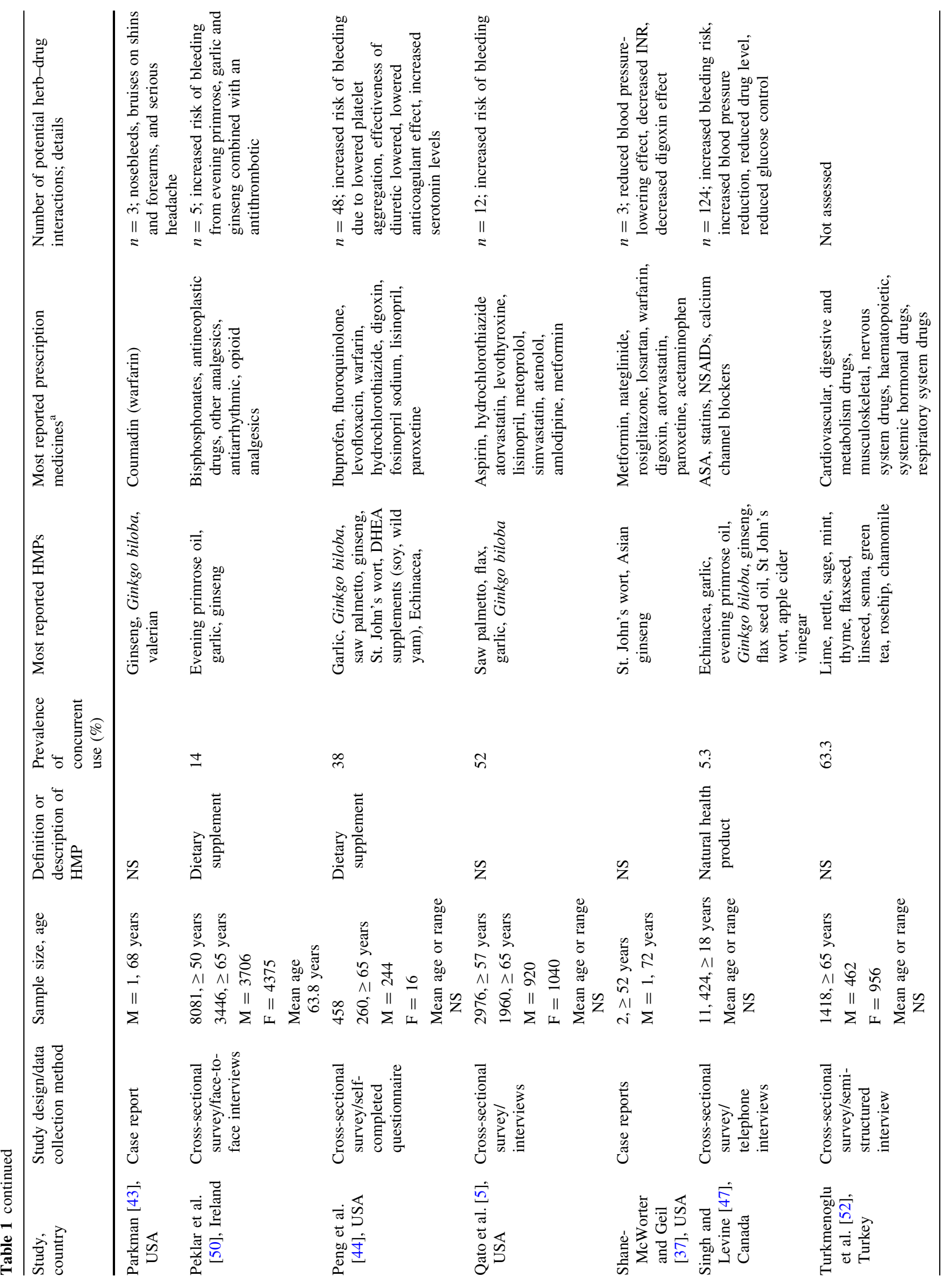




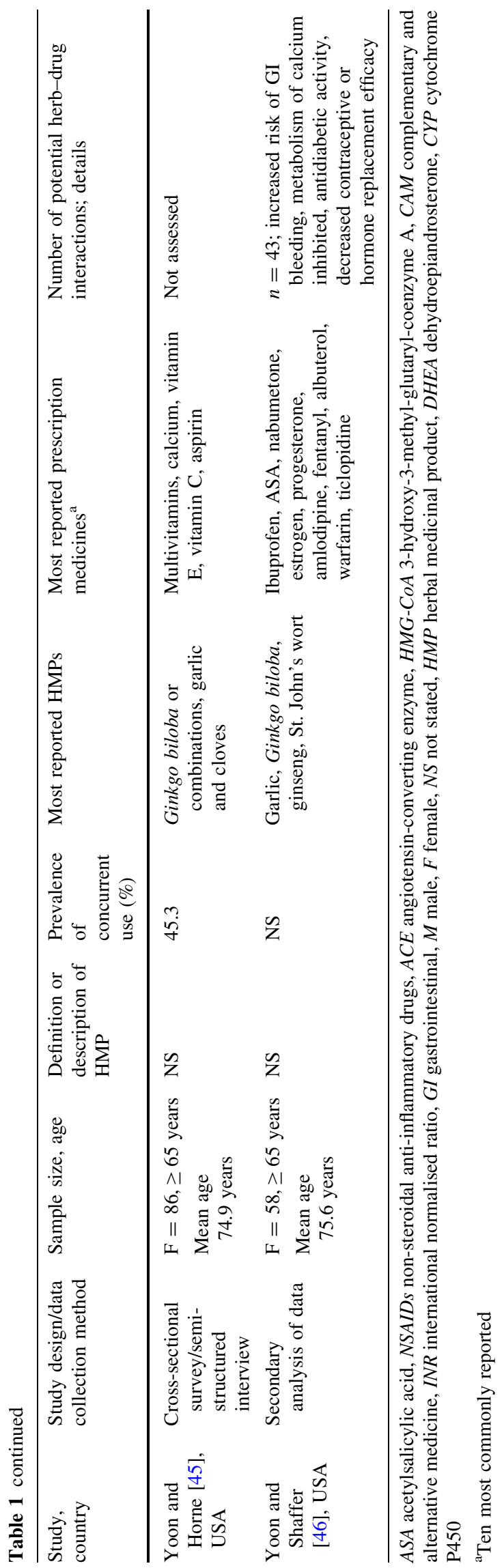

other medications using semi-structured interviews [17, 36, 38, 40, 45, 47, 52, 53]; others interviewed older people, and then checked and recorded their medications [5, 17, 42, 48, 50, 54]. Self-completed questionnaires were adopted in five studies [41, 42, 44, 49, 51], with participants self-reporting on the questionnaire all the medicines they were taking. Three studies $[16,46,54]$ were secondary analyses of data from previous research and three were case reports [37, 39, 43] of possible interactions between herbal dietary supplements and prescription medicines. Only four studies have been published in the last 5 years [36, 50-52]. Seventeen studies were published between 2000 and 2010, and one case report was published in 1999 [39].

The 22 studies included in this review had a total of 18,399 participants aged 65 years or over. The average age of participants ranged from 63 to 78 years, and the number of participants ranged from one (case report) to 5052. Only in ten studies was the focus on those aged 65 years or older, with the other studies conducted among the general population aged $\geq 18$ years, but data for participants aged $\geq 65$ years could be extracted.

Participants were predominantly females in 12 studies, varying between $51 \%$ [17] and $100 \%$ [46, 55]. Male participants were the majority in five studies [16, 41-44]. The number of males and females in the different age categories were not specified in four studies [38, 47, 51, 53]. One study each was conducted among older adults in hospitals [52] and nursing homes [36]. The remaining studies were conducted among general populations (i.e. communitydwelling older adults) $[5,16,17,37-41,46,47$, 49-51, 53, 55], outpatients of memory clinics [42, 48], emergency department [43] and veteran centre [44].

We ensured only studies that actually evaluated HMPs were included by looking at the definition where provided and the herbal medications reported. However, no consistent term exists for HMPs and different terms are used in different countries. For example, in Canada, HMPs are referred to as natural health products (NHPs), i.e. "Substances or combination of substances consisting of molecules and elements found in nature and homeopathic preparations sold in dosage forms for the purpose of maintaining or improving health, and treating or preventing diseases/conditions, and includes herbal medicines, vitamins and minerals" [56; p. 2]. Both Canadian studies included in this review [47, 48] used the term "natural health products'. Only one study from the US [38] used 'herbs/natural products', but excluded vitamins and minerals.

Elmer et al. [54] used the term complementary and alternative medicine (CAM) products, defined as "products such as herbal (botanical) products or non-botanical dietary supplements (e.g. glucosamine) excluding vitamins and 
minerals". Five studies $[17,39,42,44,50]$ used the definition of dietary supplement according to Directive 2002/46/EC of the European Parliament and of the Council, 2002 [26], i.e. "potentially any product intended for ingestion as a supplement to regular diet, including vitamins or minerals (at any dose level), herbal products, and nutraceuticals". Twelve studies [5, 16, 37, 40, 41, 43, 46, 49, 51-53, 55] provided no definition or an explanation of HMP. All potentially eligible studies were therefore individually screened to ensure they met this inclusion criterion independent of the definition used.

\subsection{Synthesis of Results}

\subsubsection{Prevalence of Concurrent Prescription Drugs and Herbal Medicinal Products (HMPs) Among Older Adults}

Fifteen studies reported prevalence of concurrent use, while no such information was provided in four articles $[16,46,49,53]$ and three were case reports where prevalence cannot be calculated [37, 39, 43]. Prevalence of concurrent use varied widely between $5.3 \%$ [47] and $88.3 \%$ [42].

Table 1 shows the most concurrently combined prescription medicines and HMPs from the included studies. The common groups of prescription medicines concurrently combined with HMPs were antihypertensive drugs, $\beta$-blockers, diuretics, antihyperlipidemic agents, anticoagulants, analgesics, antihistamines, antidiabetics, antidepressants and statins.

The most commonly used HMPs as reported in the included studies were Ginkgo (Ginkgo biloba), garlic (Allium sativum), Ginseng (Panax ginseng), St John's wort (Hypericum perforatum), Echinacea (Echinacea purpurea), saw palmetto (Serenoa repens), evening primrose oil (Oenothera biennis) and ginger (Zingiber officinale). In some studies, non-herbal dietary or nutritional supplements $[37,41,42,47,49,50]$, vitamins and minerals $[17,42,44,50]$ and OTC conventional medicines [40, 48, 53, 54] were also concurrently used by participants in addition to prescription drugs and HMPs. In one study [42], $82.5 \%$ of participants receiving prescription medicines also used at least one non-herbal dietary supplement, while $54.5 \%$ used three or more.

\subsubsection{Potential Interactions and Safety Issues}

Potential interactions from reported combinations of prescription drugs and HMPs were evaluated using different methods. Some studies used a combination of two or more of the following methods: review of possible interactions from previously published clinical data, case reports and textbooks [16, 41, 42, 47, 51, 54], and comprehensive online databases such as Micromedex (https://www. micromedexsolutions.com), Natural Medicines (https:// naturalmedicines.therapeuticresearch.com/, formerly Natural Standard) and Stockley's Drug Interactions (http:// www.pharmpress.com/product/MC_STOCK/stockleysdrug-interactions) $[5,40,42,44,46,50]$.

Due to how data were presented in two studies [49, 51], it was not possible to extract potential interactions for participants aged $\geq 65$ years. No evaluation of potential interactions was done in five studies [17, 42, 45, 52, 53], while a total of 1010 individual interactions or potential interactions were reported in 15 studies. The potential risks of bleeding due to the use of Ginkgo biloba, garlic or ginseng with aspirin and warfarin were the most reported $[5,36,37,41,43,44,46,48,50,54]$, or with other antithrombotic drugs [50]. Other interactions reported included the risk of decreased international normalised ratio (INR) [16, 37], alterations in either blood glucose or blood pressure [40], nausea and dizziness [39], anxiety [16], headaches [39, 43], restlessness and irritability [16]. An important and risky mode of herb-drug interaction is the inhibition of cytochrome P450 3A4 substrates (e.g. atorvastatin, simvastatin, amlodipine, verapamil) by garlic, Ginkgo biloba, Echinacea and St John's wort [38]. For example, St. John's wort could reduce the blood pressurelowering effect of losartan, or decrease the effects of digoxin [37].

Interactions were rated by the authors as 'major or high risk', 'moderate' or 'minor'. The majority of potential interactions reported in the included studies were minor and of unknown clinical significance or uncertain risk for an adverse interaction [16, 54]. These interactions were cited in the literature based only on theoretical evidence [47]. Potential major herb-drug interactions reported were between non-steroidal anti-inflammatory drugs (NSAIDs) and Ginkgo biloba, resulting in an increased risk of gastrointestinal bleeds due to decreased platelet aggregation [46]. Other major interactions occurred between drugs and non-herbal supplements [50], or involved the use of nonprescription drugs [5].

\subsubsection{Concurrent Use and Associated Factors}

The majority of studies included in this review did not assess concurrent use with demographic or clinical variables. For the 11 studies that assessed demographic or clinical factors [5, 17, 38, 45, 47, 49-54], the following can be summarised:

3.3.3.1 Ethnicity Only one study assessed the differences in concurrent use between different ethnic groups. African Americans used significantly more garlic $(p=0.003)$, 
although no significant difference was observed in the use of ginseng or Ginkgo biloba between African Americans and White participants [54].

3.3.3.2 Sex and Age An important sex difference in medication use among older adults was observed in seven studies [5, 17, 38, 47, 49-51]. Women used more herbal supplements than men [5, 49], while a significantly higher prevalence of use of five or more prescription medications among women aged 57 through 64 years was reported in two studies [5, 17]. Consequently, more women than men concurrently use HMPs with prescription medicines [5, 38, 50, 51]. Qato et al. [5] found up to $60 \%$ of women in the oldest age groups used prescription medications in combination with herbal dietary supplements. Furthermore, increased odds for a co-user to be female (34 vs. $18 \%$, $p=0.001$ ) and older (more than one in every three were older than 50 years of age) was also confirmed by Djuv et al. [51].

Two studies [45, 50] found no association between age and concurrent use. Singh and Levine [47] reported that older users who combined prescriptions with NHPs, and females, were more likely to have potential interactions than males who combined prescriptions with NHPs (63 vs. $48 \%)$.

\subsubsection{Disease State or Clinical Condition Five studies} [47, 50-53] compared concurrent use with disease state or clinical conditions. Herbal product use was slightly higher among participants who experienced ongoing health problems (31.1\%) than healthy older adults $(24.9 \%)$, although the difference was not significant. Consequently, herbal product use was significantly higher among participants who reported continuous drug use compared with those who did not use any drugs [52]. Increased levels of co-use were associated with the use of analgesics or a dermatological drug [51], and chronic diseases were associated with an increased likelihood of concurrent prescription and supplement use [50]. High blood pressure and diabetes were also strongly associated with potential interaction [47]. However, Delgoda et al. [53] found no significant association between concurrent herb-drug use and a participant's disease.

3.3.3.4 Education and Household Income Only four studies [47, 50, 52, 53] assessed the educational level or household income of participants with concurrent use. Concurrent herb-drug use was greater among individuals who had an education no higher than secondary level $[50,53]$, and higher education was associated with a lower probability of potential interaction [47]. Therefore, compared with post-secondary graduates, participants with less than a high-school education were $70 \%$ more likely to exhibit at least one potential interaction [47].

The prevalence of concurrent herb-drug use was also greater among individuals from households with a lower household income or with no form of health insurance [53]. Having private medical insurance was associated with an increased likelihood of using HMPs [50]; however, Turkmenoglu et al. [52] found no significant associations between HMP use and income.

\subsubsection{Disclosure of HMP Use to Healthcare Profes-} sionals Only six of the included studies asked participants if the use of HMPs was disclosed to their doctors or other healthcare professionals [41, 44, 45, 51-53]. No distinct trend was observed among the six studies and disclosure varied widely between $12 \%$ [52] and 78\% [44]. A study of 1418 older adults [52] reported that $42.2 \%$ ( $n=180$ ) of concurrent users believed herbal products were not harmful and therefore did not need to discuss these with their healthcare providers. Although 51 participants (12\%) always reported herbal use to their physician, $40 \%$ ( $n=169$ ) would only disclose herbal product use to healthcare providers if asked, and 2.8\% $(n=12)$ would only disclose herbal product use if they had a problem. In another study [44], 78\% of participants reported HMP use, although 58 of the 99 concurrent users said they were not questioned by healthcare practitioners on their HMP use. Approximately $64 \%$ of co-users $(n=18)$ of HMPs and prescription drugs disclosed use in one study [41], while in another study, almost $80 \%$ of users of HMPs did not disclose use [51].

\subsubsection{Expenditure on HMPs and Satisfaction Only two} studies conducted in the US in 2002 and 2004 [41, 44], respectively, considered the cost or resources spent on HMPs by older adults. The majority of concurrent users (64 and $83 \%$ ) spent $\$ 25$ or less on HMPs per month, approximately $15 \%$ spent between $\$ 25$ and $\$ 50$ per month [44], and only 3 of 28 (11\%) [41] and 1 of 99 (1\%) [44] concurrent users spent more than $\$ 100$ per month on HMPs.

\subsection{Quality Appraisal}

Considering the paucity of research in this area, a cut-off score of 4 was accepted for each JBI checklist to ensure there were sufficient studies to review while maintaining the strength of methodological quality. Typically, research in this area is not randomised; a score of 7 and above indicated high quality, while a score of 4-6 indicated moderate quality. All 22 studies were of sufficient quality and were included in this review. 


\section{Discussion}

This systematic review included a total of 22 studies that investigated concurrent use of prescription medicines with HMPs. The majority of studies were conducted in the US, with only four of the studies being conducted in the last 5 years. It can be concluded from the results presented that the prevalence of concurrent prescription and HMP use among older adults is substantial. The most commonly combined prescription drugs by older adults are antihypertensive drugs, $\beta$-blockers, diuretics, antihyperlipidemic agents, anticoagulants, analgesics, antihistamines, antidiabetics, antidepressants and statins. And the HMPs most commonly combined include Ginkgo biloba, garlic, ginseng, St John's wort, Echinacea, saw palmetto, evening primrose and ginger. Furthermore, there are demographic and clinical factors associated with concurrent prescription and HMP use. Women, as well as individuals in the oldest age groups, with chronic conditions, less than a high-school education and receiving a low income, are more likely to be concurrent users. The most common potential interaction was the risk of bleeding from combinations of Ginkgo biloba, garlic or ginseng with aspirin and warfarin, all of which are frequently used by older adults.

The included studies varied greatly in terms of participants, products and outcome measures. Generic terms such as 'elderly' or 'older persons' are commonly used [57], but there is no concrete definition of these terms. While ageing is an inevitable process measured by chronological age, its impact varies across populations [58]; therefore, different definitions and chronological age are adopted in clinical studies. While some authors regarded 'older adults' or 'elderly' as those aged 65 years and older, others used the cut-off point of 60 years, or even 75 years, which affected both how participants were grouped and the synthesis of data. Furthermore, many studies looked at adult populations including 'older adults' or 'elderly', but did not, or only partially, report results separately for this age group. In the latter case, only results that were clearly reported for adults aged 65 years and older were included in our analysis. We therefore had to exclude a number of potentially relevant articles due to either a lack of definition or separate reporting.

The heterogeneity in definitions adopted for HMPs and the inconsistencies on what is included as an HMP demonstrates the lack of precision around what may or may not be seen as an HMP. While one study [54] adopted the term 'complementary and alternative medicine', excluding vitamins and minerals, other studies adopted the terms 'natural health product' and 'dietary supplements', including both vitamins and minerals. Moreover, many did not differentiate between HMPs and dietary supplements, but rather included all types of medications, including vitamins, minerals, and herbal and non-herbal dietary supplements. We only included studies of HMPs that were explicitly named in the Results section. This variation did not allow for comparisons across studies to be conducted, and also blurred what might be seen as nutritional interventions to improve overall health and those that are used explicitly for medicinal purposes to address specific medical conditions.

The prevalence of concurrent prescriptions and HMP use among adults aged 65 years and older ranged from 5.3 to $88.3 \%$. Several factors might explain the discrepancies in the prevalence of concurrent use reported in studies included in this review. First, variation in the range of prevalence reflected the different definitions, types of HMPs assessed, and participants. Second, many of the studies relied on patient recall of the prescription and herbal medicines they use, possibly resulting in recall bias. In some studies [5, 17, 42, 48, 50, 54], participants took bottles and containers of medicines they were taking along to interviews, for documentation by the research teams.

One of the outliers, an analysis of the 2000-2001 Canadian National Population Health Survey, reported only 5.3\% concurrent use of NHPs with prescription medications [47]. This difference in prevalence may be explained by underreporting or recall bias due to how the data were collected. Participants were asked for the medications and NHPs used in the previous $24 \mathrm{~h}$. This is unusual compared with other surveys on this topic where current and previous use over 2 weeks [42] and up to 12 months was requested [38, 41, 49]. Therefore, the data may have revealed only a percentage of respondents exposed to an NHP during a limited time period. In addition, herbs and other NHPs are widely used in a variety of foods, beverages, and multivitamin supplements, but because these were not specifically asked about in the survey, it is possible that their use was not reported. Therefore, the true prevalence of concurrent prescriptionNHP interactions in the study population may be higher than reported.

The other outlier is a Spanish study that reported a prevalence of concurrent use of $88.3 \%$. The study assessed both commercially prepared HMPs and home remedies concurrently used with prescription medicines among community-dwelling older adults and those resident in care homes. All medicinal plants, including teas and spices, widely consumed in Spain were included in the analysis, which may have contributed to the high prevalence rate recorded in this study.

Three [5, 42, 45] of the five studies [5, 36, 42, 45, 52] with the highest prevalence rates, ranging between 45.3 and $83 \%$, were conducted in the US. These high prevalence rates could be due to the healthcare system or the 
sociocultural characteristics of the location where research was conducted. For the American studies, patients potentially used HMPs and non-prescription drugs for prevention or self-treatment [45] as alternatives to expensive medical consultations and prescription drugs. Second, only one [42] of the five studies provided a definition of what is regarded as an HMP. Considering the inconsistencies in what HMPs include, it is possible that other non-herbal dietary products were considered.

Demographic characteristics, as well as health status, have been associated with the use of herbal medicines and natural products. Sex, age, ethnicity and health status may result in greater use of herbs and natural products [38]. Although only $50 \%$ of the studies included in this review compared demographic characteristics and health status with concurrent use, the results confirm earlier findings [11] that the use of herbal medicines varies widely between countries and ethnic groups. For example, the two Canadian studies [47, 48] reported lower rates compared with studies from the US. In addition, the rate of combining prescription medications and dietary supplements was higher among women than men across all age groups $[5,17]$. These trends were also reported in earlier studies $[59,60]$.

Sex differences in concurrent use among older adults may be explained by the higher prevalence of chronic conditions among women compared with men [61]. Concurrent use was greater among older adults from households with a lower household income, no health insurance and no post-secondary education, which may be due to the type of healthcare system, i.e. paid for or free at the point of delivery. It is therefore reasonable to assume that in such countries, participants may rely more on HMPs, or use them as alternatives to expensive medical consultations.

Although there is increased awareness of interactions between conventional drugs and HMPs, the lack of agreement about how to identify HMPs or rigorous clinical evidence hinders researchers, clinicians and consumers in making informed decisions about safe combinations of conventional drugs and HMPs [62]. The majority of the evidence on herb-drug interactions is from case reports. Arguably, the scarcity and poor quality of primary research may mean that interactions of serious consequences associated with concurrent use of HMPs are unknown and unrecognised [63]. The evidence from this review would suggest that there is potential for harm.

There is a potentially high rate of unreported use of HMPs among older adults. Only $28 \%$ of included studies asked participants if the use of HMPs was disclosed to healthcare professionals. Our findings confirm previous research [64-66] that only approximately one-third of HMP users disclose use to healthcare professionals. Disclosure of herbal medicine use is crucial to avoiding herb- drug interactions and non-adherence to prescription medications. The reasons for non-disclosure of HMP use, as reported in this review and confirmed by other studies, includes a perceived negative attitude of clinicians to complementary medicine use [66, 67], clinicians do not ask [66, 68, 69], and the notion that HMPs are 'harmless' [68].

\subsection{Limitations}

The main limitation of this review is the heterogeneity or non-definition of HMPs in available studies, which prevented a meta-analysis. Second, we had to exclude a large number of studies because either the use of HMPs was unclear or results reported were not age-specific to enable us to extract data for subjects aged $\geq 65$ years. Finally, only four of the included studies were published in the last 5 years [36, 50-52]; 17 were published between 2000 and 2010, and one case report was published in 1999 [39]. The increasing use of HMPs worldwide could mean that the review underestimates the range and scale of the issues.

\subsection{Implications for Practice}

Evidence from this review indicates that a large number of older adults concurrently use prescription drugs and HMPs, and the majority do not disclose this to healthcare practitioners. However, the findings do demonstrate that certain combinations of prescription drugs and HMPs can have serious consequences. Therefore a better understanding of the extent and manner in which older adults combine prescription drugs and HMPs in their health regimens, and the associated risks, is important for healthcare practitioners.

\section{Conclusions}

The prevalence of concurrent use of prescription drugs and HMPs by older adults is generally substantial, although variations in the extent of use are reported. These variations can be explained by methodological factors, including definition of HMPs, participant selection, sociodemographic factors and differences in healthcare systems. Concurrent use of prescription drugs and HMPs is associated with risks, some with potentially serious consequences. The most reported interactions in older adults were risk of bleeding due to the use of Ginkgo biloba, garlic or ginseng in combination with aspirin and warfarin or other antithrombotic drugs. Underreporting is substantial and adds to the problem, considering that in most countries there are no appropriate safeguards to minimise the potential harm. By identifying the most commonly used combinations, healthcare professionals, including 
pharmacists, can be informed on how to appropriately identify and manage patients at risk. It also highlights the need for targeted patient information provided by healthcare professionals and pharmacists as part of routine consultations. Further research is needed to explore why older people use HMPs alongside their prescribed medication, and how their decisions regarding preferred treatments can be documented and discussed by prescribing clinicians, in order to identify and manage the potential risk of herbdrug interactions.

Acknowledgements The authors wish to thank José J. Mira for extracting data from the Spanish article.

Author contributions This systematic review is part of TA's Ph.D. in Health Research at the University of Hertfordshire. The review was her original idea, with support and advice from BW and CG in the development and refinement of the review questions. LW designed the search strategy and conducted the literature searches. TA and BW screened and selected the relevant studies, extracted the data, performed the quality appraisal and synthesised the data. TA wrote the first draft of the article, and BW and CG contributed to the subsequent writing of the article. All authors reviewed and agreed with the results and conclusions of the review.

\section{Compliance with Ethical Standards}

Funding No sources of funding were used to assist in the preparation of this article.

Conflicts of interest Taofikat Agbabiaka, Barbara Wider, Leala Watson and Claire Goodman declare that they have no conflicts of interest relevant to the content of this review.

Open Access This article is distributed under the terms of the Creative Commons Attribution-NonCommercial 4.0 International License (http://creativecommons.org/licenses/by-nc/4.0/), which permits any noncommercial use, distribution, and reproduction in any medium, provided you give appropriate credit to the original author(s) and the source, provide a link to the Creative Commons license, and indicate if changes were made.

\section{References}

1. World Health Organization. 10 facts on ageing and the life course. 2012. http://www.who.int/features/factfiles/ageing/ ageing_facts/en/index.html. Accessed 20 April 2017.

2. Office for National Statistics. National population projections for the UK- 2014-based. 2015. https://www.ons.gov.uk/people populationandcommunity/populationandmigration/population projections/bulletins/nationalpopulationprojections/2015-1029\#changing-age-structure. Accessed 10 March 2017.

3. Cherubini A, Corsonello A, Lattanzio F. Underprescription of beneficial medicines in older people. Drugs Aging. 2012;29(6): 463-75.

4. Nyborg G, Straand J, Brekke M. Inappropriate prescribing for the elderly-a modern epidemic? Eur J Clin Pharmacol. 2012;68(7):1085-94.

5. Qato DM, Alexander GC, Conti RM, Johnson M, Schumm P, Lindau ST. Use of prescription and over-the-counter medications and dietary supplements among older adults in the United States. JAMA. 2008;300(24):2867-78.

6. Qato DM, Wilder J, Schumm LP, Gillet V, Alexander GC. Changes in prescription and over-the-counter medication and dietary supplement use among older adults in the United States, 2005 vs 2011. JAMA Intern Med. 2016;176(4):473-82.

7. National Council on Patient Information and Education. Fact sheet: medicine use and older adults. 2010. http://www. mustforseniors.org/facts.jsp. Accessed 12 Jan 2017.

8. Jerez-Roig J, Medeiros LF, Silva VA, Bezerra CL, Cavalcante LA, Piuvezam G, et al. Prevalence of self-medication and associated factors in an elderly population: a systematic review. Drugs Aging. 2014;31(12):883-96.

9. Vacas Rodilla E, Castella Daga I, Sanchez Giralt M, Pujol Algue A, Pallares Comalada MC, Balague Corbera M. Self-medication and the elderly. The reality of the home medicine cabinet. Aten Primaria. 2009;41(5):269-74.

10. Bruno JJ, Ellis JJ. Herbal use among US elderly: 2002 National Health Interview Survey. Ann Pharmacother. 2005;39(4):643-8.

11. Raji MA, Kuo Y-F, Al Snih S, Sharaf BM, Loera JA. Ethnic differences in herb and vitamin/mineral use in the elderly. Ann Pharmacother. 2005;39(6):1019-23.

12. Marinac JS, Buchinger CL, Godfrey LA, Wooten JM, Sun C, Willsie SK. Herbal products and dietary supplements: a survey of use, attitudes, and knowledge among older adults. J Am Osteopath Assoc. 2007;107(1):13-23.

13. Gonzalez-Stuart A. Herbal product use by older adults. Maturitas. 2011;68(1):52-5.

14. de Souza Silva JE, Souza CAS, da Silva TB, Gomes IA, de Carvalho Brito G, de Souza Araújo AA, et al. Use of herbal medicines by elderly patients: a systematic review. Arch Gerontol Geriatr. 2014;59(2):227-33.

15. Izzo AA, Ernst E. Interactions between herbal medicines and prescribed drugs: a systematic review. Drugs. 2001;61(15):2163-75.

16. Izzo AA, Ernst E. Interactions between herbal medicines and prescribed drugs: an updated systematic review. Drugs. 2009;69(13):1777-98.

17. Kaufman DW, Kelly JP, Rosenberg L, Anderson TE, Mitchell AA. Recent patterns of medication use in the ambulatory adult population of the United States: the Slone survey. JAMA. 2002;287(3):337-44.

18. Leiss W, Méan M, Limacher A, Righini M, Jaeger K, Beer H-J, et al. Polypharmacy is associated with an increased risk of bleeding in elderly patients with venous thromboembolism. J Gen Intern Med. 2015;30(1):17-24.

19. Hilmer S, Gnjidic D. The effects of polypharmacy in older adults. Clin Pharmacol Ther. 2009;85(1):86-8.

20. Davies EC, Green CF, Taylor S, Williamson PR, Mottram DR, Pirmohamed M. Adverse drug reactions in hospital in-patients: a prospective analysis of 3695 patient-episodes. PLoS One. 2009;4(2):e4439.

21. Mangoni AA, Jackson SH. Age-related changes in pharmacokinetics and pharmacodynamics: basic principles and practical applications. Br J Clin Pharmacol. 2004;57(1):6-14.

22. Sultana J, Cutroneo P, Trifirò G. Clinical and economic burden of adverse drug reactions. J Pharmacol Pharmacother. 2013;4(Suppl 1):S73-7.

23. Health and Social Care Information Centre. Prescriptions dispensed in the community: England 2004-14. 2015. http://content. digital.nhs.uk/catalogue/PUB17644/pres-disp-com-eng-2004-14rep.pdf. Accessed 25 Sept 2016.

24. MHRA, Ipsos MORI. Public perception of herbal medicines. London: MHRA; 2009.

25. Lynch N, Berry D. Differences in perceived risks and benefits of herbal, over-the-counter conventional, and prescribed 
conventional, medicines, and the implications of this for the safe and effective use of herbal products. Complement Ther Med. 2007;15(2):84-91.

26. Commission of the European Communities. Amended proposal for a Directive of the European Parliament and of the Council amending the directive 2001/83/EC as regards traditional herbal medicinal products. Brussels: European Commission; 2003. p. 67.

27. Fugh-Berman A. Herb-drug interactions. Lancet. 2000;355(9198):134-8.

28. Williamson EM. Synergy and other interactions in phytomedicines. Phytomedicine. 2001;8(5):401-9.

29. Williamson EM, Driver S, Baxter K, editors. Stockley's Herbal Medicines Interactions. 2nd ed. London: Pharmaceutical Press; 2013.

30. Agbabiaka T, Wider B, Watson LK, Goodman C. Concurrent use of prescription drugs and herbal medicinal products in older adults: a systematic review protocol. Syst Rev. 2016;5(1):1-5.

31. The Cochrane Collaboration. Cochrane handbook for systematic reviews of interventions, version 5.1.0. 2011. www.handbook. cochrane.org. Accessed 12 June 2015.

32. Shamseer L, Moher D, Clarke M, Ghersi D, Liberati A, Petticrew M. Preferred reporting items for systematic review and metaanalysis protocols (PRISMA-P) 2015: elaboration and explanation. BMJ. 2015;350:g7647.

33. Munn Z, Moola S, Lisy K, Riitano D, Tufanaru C. Methodological guidance for systematic reviews of observational epidemiological studies reporting prevalence and cumulative incidence data. Int J Evid Based Healthc. 2015;13(3):147-53.

34. The Joanna Briggs Institute. Joanna Briggs Institute reviewers' manual. 2016th ed. Adelaide: The Joanna Briggs Institute; 2016.

35. Kavanagh J, Campbell F, Harden A, Thomas J. Mixed methods synthesis: a worked example. In: Hannes K, Lockwood C, editors. Synthesizing qualitative research: choosing the right approach. Chichester, UK: Wiley; 2011. p. 113-36.

36. Batanero-Hernán C, Guinea-López M, García-Jiménez E, Rodríguez-Chamorro M. Análisis del consumo simultáneo de medicamentos y plantas medicinales en población española mayor de 65 años. Pharmaceutical Care España. 2017;19(2):69-79.

37. Shane-McWhorter L, Geil P. Interactions between complementary therapies or nutrition supplements and conventional medications. Diabetes Spectr. 2002;15(4):262-6.

38. Blalock SJ, Gregory PJ, Patel RA, Norton LL, Callahan LF, Jordan JM. Factors associated with potential medicationherb/natural product interactions in a rural community. Altern Ther Health Med. 2009;15(5):26-34

39. Lantz MS, Buchalter E, Giambanco V. St. John's wort and antidepressant drug interactions in the elderly. J Geriatr Psychiatry Neurol. 1999;12(1):7-10.

40. Loya AM, Gonzalez-Stuart A, Rivera JO. Prevalence of polypharmacy, polyherbacy, nutritional supplement use and potential product interactions among older adults living on the United States-Mexico border: a descriptive, questionnaire-based study. Drugs Aging. 2009;26(5):423-36.

41. Ly J, Percy L, Dhanani S. Use of dietary supplements and their interactions with prescription drugs in the elderly. Am J Health Syst Pharm. 2002;59(18):1759-63.

42. Nahin RL, Pecha M, Welmerink DB, Sink K, DeKosky ST, Fitzpatrick AL. Concomitant use of prescription drugs and dietary supplements in ambulatory elderly people. J Am Geriatr Soc. 2009;57(7):1197-206.

43. Parkman CA. Polypharmacy, herbal therapies, and elders. Case Manager. 2001;12(4):32-4.

44. Peng CC, Glassman PA, Trilli LE, Hayes-Hunter J, Good CB. Incidence and severity of potential drug-dietary supplement interactions in primary care patients: an exploratory study of 2 outpatient practices. Arch Intern Med. 2004;164(6):630-6.

45. Yoon S, Horne C. Herbal products and conventional medicines used by community-residing older women. J Adv Nurs. 2001;33(1):51-9.

46. Yoon SL, Schaffer SD. Herbal, prescribed, and over-the-counter drug use in older women: prevalence of drug interactions. Geriatr Nurs. 2006;27(2):118-29.

47. Singh SR, Levine MA. Potential interactions between pharmaceuticals and natural health products in Canada. J Clin Pharmacol. 2007;47(2):249-58.

48. Dergal JM, Gold JL, Laxer DA, Lee MS, Binns MA, Lanctot KL, et al. Potential interactions between herbal medicines and conventional drug therapies used by older adults attending a memory clinic. Drugs Aging. 2002;19(11):879-86.

49. Canter PH, Ernst E. Herbal supplement use by persons aged over 50 years in Britain: frequently used herbs, concomitant use of herbs, nutritional supplements and prescription drugs, rate of informing doctors and potential for negative interactions. Drugs Aging. 2004;21(9):597-605.

50. Peklar J, Henman MC, Kos M, Richardson K, Kenny RA. Concurrent use of drugs and supplements in a community-dwelling population aged 50 years or more: potential benefits and risks. Drugs Aging. 2014;31(7):527-40.

51. Djuv A, Nilsen OG, Steinsbekk A. The co-use of conventional drugs and herbs among patients in Norwegian general practice: a cross-sectional study. BMC Complement Altern Med. 2013;13(1):295.

52. Turkmenoglu FP, Kutsal YG, Dolgun AB, Diker Y, Baydar T. Evaluation of herbal product use and possible herb-drug interactions in Turkish elderly. Complement Ther Clin Pract. 2016;23:46-51.

53. Delgoda R, Younger N, Barrett C, Braithwaite J, Davis D. The prevalence of herbs use in conjunction with conventional medicines in Jamaica. Complement Ther Med. 2010;18(1):13-20.

54. Elmer GW, Lafferty WE, Tyree PT, Lind BK. Potential interactions between complementary/alternative products and conventional medicines in a Medicare population. Ann Pharmacother. 2007;41(10):1617-24.

55. Yoon SJ. Nearly half of all women 65 and older use herbal products to feel better, but don't tell their doctors. Fla Nurse. 2001;49(1):27.

56. Health Canada. Natural Health Products Regulation. 2003. http:// laws-lois.justice.gc.ca/PDF/SOR-2003-196.pdf. Accessed 10 April 2017.

57. Singh S, Bajorek B. Defining 'elderly' in clinical practice guidelines for pharmacotherapy. Pharm Pract (Granada). 2014;12(4):489.

58. Levine ME. Modeling the rate of senescence: can estimated biological age predict mortality more accurately than chronological age? J Gerontol A Biol Sci Med Sci. 2013;68(6):667-74.

59. Radimer K, Bindewald B, Hughes J, Ervin B, Swanson C, Picciano MF. Dietary supplement use by US adults: data from the National Health and Nutrition Examination Survey, 1999-2000. Am J Epidemiol. 2004;160(4):339-49.

60. Gardiner P, Graham RE, Legedza AT, Eisenberg DM, Phillips RS. Factors associated with dietary supplement use among prescription medication users. Arch Intern Med. 2006;166(18):1968-74.

61. Tsang A, Von Korff M, Lee S, Alonso J, Karam E, Angermeyer $\mathrm{MC}$, et al. Common chronic pain conditions in developed and developing countries: gender and age differences and comorbidity with depression-anxiety disorders. J Pain. 2008;9(10):883-91.

62. Zhang X-L, Chen M, Zhu L-L, Zhou Q. Therapeutic risk and benefits of concomitantly using herbal medicines and 
conventional medicines: from the perspectives of evidence based on randomized controlled trials and clinical risk management. Evid Based Complement Alternat Med. 2017;2017:9296404.

63. Posadzki P, Watson L, Ernst E. Herb-drug interactions: an overview of systematic reviews. $\mathrm{Br} \mathrm{J}$ Clin Pharmacol. 2013;75(3):603-18.

64. Kennedy J, Wang C-C, Wu C-H. Patient disclosure about herb and supplement use among adults in the US. Evid Based Complement Alternat Med. 2008;5(4):451-6.

65. Mehta DH, Gardiner PM, Phillips RS, McCarthy EP. Herbal and dietary supplement disclosure to health care providers by individuals with chronic conditions. J Altern Complement Med. 2008;14(10):1263-9.
66. Robinson A, McGrail M. Disclosure of CAM use to medical practitioners: a review of qualitative and quantitative studies. Complement Ther Med. 2004;12(2):90-8.

67. Samuels N, Zisk-Rony RY, Zevin S, Becker EL, Yinnon AM, Oberbaum M. Use of non-vitamin, non-mineral (NVNM) supplements by hospitalized internal medicine patients and doctorpatient communication. Patient Educ Couns. 2012;89(3):392-8.

68. Vickers KA, Jolly KB, Greenfield SM. Herbal medicine: women's views, knowledge and interaction with doctors: a qualitative study. BMC Complement Altern Med. 2006;6(1):40.

69. Howell L, Kochhar K, Saywell R, Zollinger T, Koehler J, Mandzuk $\mathrm{C}$, et al. Use of herbal remedies by Hispanic patients: do they inform their physician? J Am Board Fam Med. 2006;19(6):566-78. 